\title{
Numerical Calibration Method for Vehicle Velocity Data from Electronic Registration Identification of Motor Vehicles Based on Mobile Edge Computing and Particle Swarm Optimization Neural Network
}

\author{
Jingfeng Yang $\mathbb{D}^{1,2}$ Zhiyong Luo, ${ }^{3}$ Nanfeng Zhang, ${ }^{4}$ Honggang Wang, ${ }^{5}$ Ming Li $\mathbb{D}$, ${ }^{6,7}$ \\ and Jinchao Xiao $(\mathbb{D})^{1,2}$ \\ ${ }^{1}$ Shenyang Institute of Automation (Guangzhou) Chinese Academy of Sciences, Guangzhou 511458, China \\ ${ }^{2}$ Shenyang Institute of Automation Chinese Academy of Sciences, Shenyang 110016, China \\ ${ }^{3}$ School of Electronics and Communication Engineering, Sun Yat-Sen University, Guangzhou 510006, China \\ ${ }^{4}$ Technical Center of Huangpu Customs District China, Guangzhou 510730, China \\ ${ }^{5}$ School of Communications and Information Engineering, Xi'an University of Posts and Telecommunications, \\ Xi'an 710061, China \\ ${ }^{6}$ South China Agricultural University, Guangzhou 510642, China \\ ${ }^{7}$ Yaz Technology Co., Ltd., Guangzhou 510630, China
}

Correspondence should be addressed to Ming Li; liming@yazcn.com and Jinchao Xiao; xiaojinchao@sia.cn

Received 20 April 2020; Revised 13 June 2020; Accepted 13 July 2020; Published 30 August 2020

Guest Editor: Yanan Li

Copyright (C) 2020 Jingfeng Yang et al. This is an open access article distributed under the Creative Commons Attribution License, which permits unrestricted use, distribution, and reproduction in any medium, provided the original work is properly cited.

In the development of technology for smart cities, the installation and deployment of electronic motor vehicle registration identification have attracted great attention in terms of smart transportation in recent years. Vehicle velocity measurement is one of the fundamental data collection efforts for motor vehicles. The velocity detection using electronic registration identification of motor vehicles is constrained by the detection algorithm, the material of the automobile windshield, the placement of the decals, the installation method of the signal reader, and the angle of the antenna. The software and hardware for electronic motor vehicle registration identification produced in the standard manner cannot meet the accuracy of velocity detection for all scenarios. Based on the actual application requirements, we propose a calibration method for the numerical output of the automobile velocity detector based on edge computing of the optimized multiple reader/writer velocity values and based on a particle swarmoptimized radial basis function (RBF) neural network. The proposed method was tested on a two-way eight-lane road, and the test results showed that it can effectively improve the accuracy of velocity detection using electronic registration identification of motor vehicles. Compared with the actual velocity, $87.12 \%$ of all the data samples had an error less than $5 \%$, and $91.76 \%$ of the data samples for vehicles in the center lane had an error less than 5\%. By calibrating the electronic vehicle velocity based on the registration identification, the accuracy of velocity detection in different application environments can be improved. Moreover, the method can establish an accurate foundation for application in traffic flow management, environmental protection, traffic congestion fee collection, and special vehicle traffic management.

\section{Introduction}

As urban development expands, the rapid increase in the number of vehicles on roads has caused various urban management problems and has led to the construction of large-scale, traffic-related infrastructures and the construction and the deployment of extensive vehicle-related facilities and equipment. These are specifically manifested in the use of various traffic sensing and road monitoring techniques and equipment including video cameras, GPS, 
geomagnetic, and radar. These devices are deployed on the road by local public security departments to ensure the safety and smooth operation of road traffic, strengthen the enforcement of traffic regulations, and ensure the intelligent control of city-level traffic. Intelligent control of traffic has become an important component of smart cities. However, video-based vehicle information recognition technology is susceptible to interference from environmental factors, such as weather, lighting, and distance from the vehicle. As a result, the effective recognition rate of video technology cannot meet the requirements of intelligent traffic management and cannot automatically identify false license plates, overlay plates, and intentionally obscured plates. Electronic registration identification of motor vehicles based on a single video image cannot satisfy the needs of smart traffic and the need to maintain a general social order [1]. To meet these needs, the application of electronic registration identification (ID) is proposed and promoted on a national level by establishing a national standard. Electronic vehicle registration identifications have been promoted in a limited number of cities and is used for intelligent parking [2], smart signal control and application [3, 4], web-linked smart vehicles [5], special vehicle passing management and special electronic registration identification of motor vehicles and control [6], and traffic operation supervision and environmental driving restrictions [7]. However, due to various technical problems, the vehicle velocity data collected using electronic vehicle registration identification still cannot serve for traffic law enforcement; this has restricted the scope of application of electronic vehicle registration identification. In terms of increasing the accuracy of collected data with spatial attributes, many researchers have conducted studies on multiobjective optimization algorithms in recent years. Grid-based methods have found good applications [8-10]. For example, Wu [11], Luo [12], and Kong [13] located robot arm movement by constructing a grid; Leong [14] maintained the diversity of solutions by constructing a grid; Knowles [8] used adaptive grids to store the obtained nondominated vectors; Yang [15] used grid technology to apply evolutionary algorithms to solve high-dimensional multiobjective optimization problems; Li [16] used grids to simultaneously characterize the convergent and distributive characteristics and proposed a multitarget particle swarm optimization algorithm based on grid sorting. The algorithm uses coordinate mapping, ordering of element optimization in the grid coordinate system, and the Euclidean distance between the element and the boundary of the approximate optimum. To improve the accuracy of the velocity data collected using electronic registration identification of motor vehicles, we combine the results of previous researchers and propose a numerical calibration method for vehicle velocity data collected by electronic registration identification of motor vehicles based on a particle swarm optimization neural network. By comparing the optimized vehicle calibration velocity and the data collected by the OBD port of the test vehicle, the reliability of the acquired velocity data is further improved.

\section{Vehicle Velocity Measurement Method Using Electronic Registration Identification of Motor Vehicles}

2.1. Installation and Connection Method for Reader/Writer of Electronic Vehicle Registration Identification. The collection of vehicle velocity data based on electronic registration identification of motor vehicles involves installing a comprehensive sensing base station at each key intersection or public security checkpoint, expressway ramp, and checkpoints at city entries. A detection coil should be installed 15 meters away from the law enforcement monitoring station or the key intersection on the main highway. To inspect the vehicles passing through the road, a gantry should be installed at a distance of 15 meters from the detection coil and an electronic registration identification of the motor vehicle device should be installed on the gantry to automatically identify the license plate of the vehicle. The reader/writer antenna installed on the gantry acquires the data from passing vehicles equipped with an electronic registration identification of motor vehicles. When the vehicle comes in contact with the coil, the detection coil emits two signals, one signal goes to the high-definition video camera and the other signal goes to the reader/writer antenna. The vehicle license plate and the electronic registration identification of the motor vehicle signal of the vehicle are captured simultaneously. After the signal is read, it is uploaded to the comprehensive sensing base station for data processing.

The system at the comprehensive sensing base station consists of a license plate recognition system and a vehicle electronic registration identification of motor vehicles decal read and write system. The system as a whole performs data collection, verification, transmission, and processing in practical applications. The overall layout is shown in Figures 1 and 2.

An appropriate location at the traffic checkpoint or the road junction is chosen to install the gantry. The induction coil is installed 15 meters from the gantry. Installed on the gantry are the electronic registration identification of motor vehicles' antenna, the high-definition video camera, and the fill-in light. Two trigger signals are sent out through the induction coil, one to the video camera and one to the vehicle electronic antenna. The antenna of the electronic registration identification of motor vehicles is connected to the read/write control of the vehicle. The video camera is connected to a front-end computer through a network or to an exchanger.

2.2. Vehicle Velocity Detection Based on Reader/Writer of Electronic Registration Identification of Motor Vehicles. The main method of using electronic registration identification of motor vehicles and readers/writers to acquire vehicle velocity data is the ultrahigh-frequency radio frequency identification technology. The radio frequency identification reader, using ultrahigh-frequency identification technology, interacts with the radio frequency electronic registration identification of motor vehicle tag installed on the wind shield of the vehicle through the ultrahigh-frequency horizontally polarized directional 


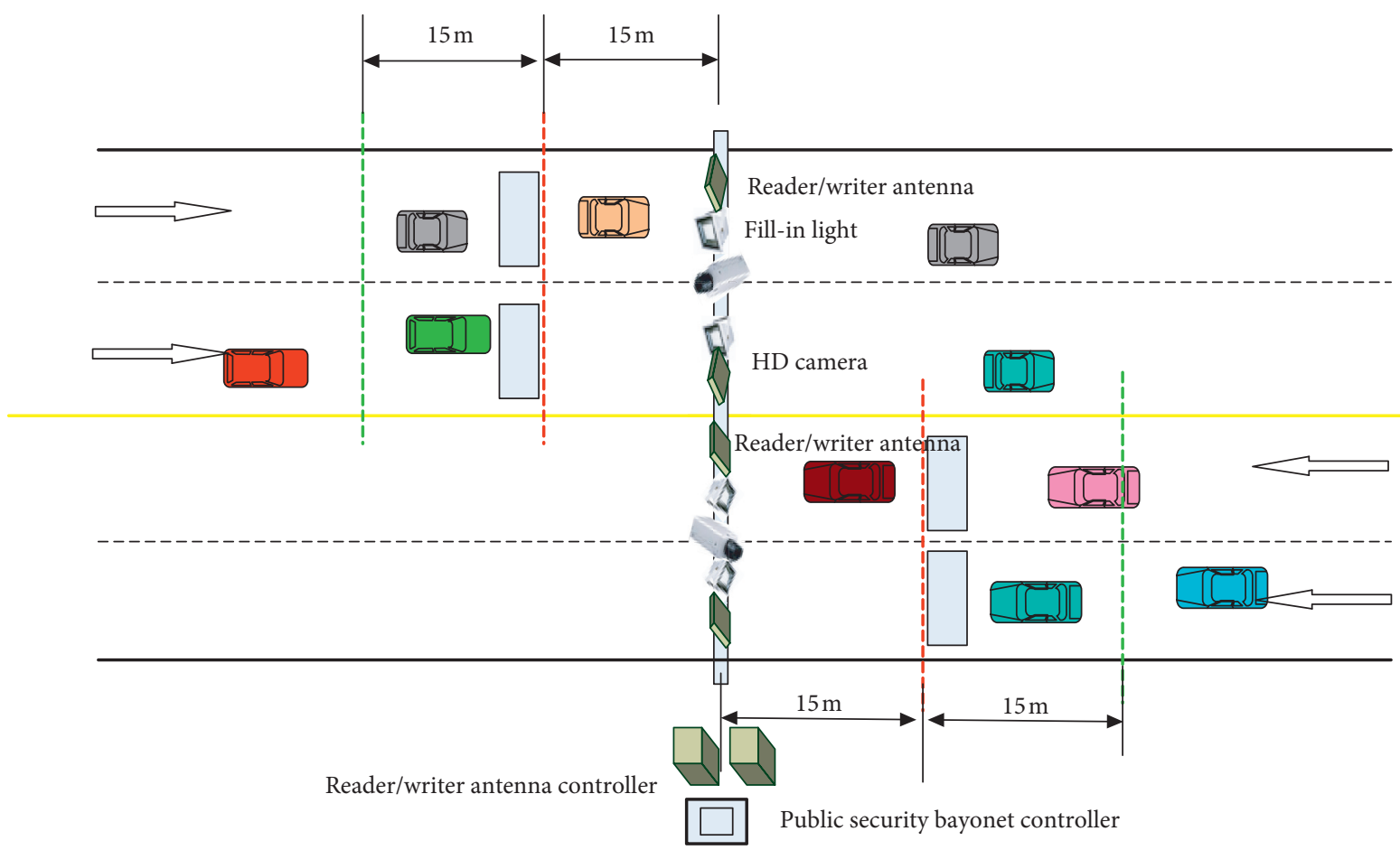

Figure 1: Overall layout diagram.

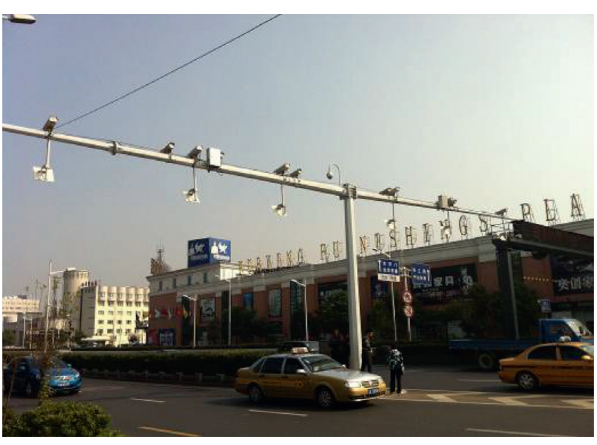

FIgURe 2: Picture of the actual installation.

antenna. The velocity of the vehicle is calculated by noting the time difference for the vehicle to go through a fixedlength identification zone. Under normal circumstances, the transmission power of the RFID reader is $30-33 \mathrm{dBm}$, and the antenna gain is $10-12 \mathrm{dBi}$. Combined with comprehensive factors such as the sensitivity of the reader and the tag, the reading and writing coverage of the RFID reader is usually between 0 and 35 meters. The steps to calculate the velocity of a vehicle using an electronic registration identification of motor vehicle tag and a reader are as follows:

Step 1. For an arbitrary vehicle shown in Figure 1, the identification record and time data are recorded as the vehicle enters the identification section of the electronic reader/writer; the same records are also recorded as the vehicle leaves the identification section.

Step 2. Interruption in the recognition record is checked to determine that the vehicle has left the identification section.
Step 3. The velocity is calculated using the formula: $V=\left(S /\left(T_{o}-T_{i}\right)\right)$,

where $V$ is the velocity with which the vehicle passes through the recognition section, $S$ is the direct distance according to the electronic registration identification of motor vehicle reader, that is, the length of the recognition section, $T_{i}$ is the time when the vehicle enters the recognition section, and $T_{o}$ is the time when the vehicle leaves the recognition section.

However, constrained by factors such as the material and angle of the vehicle's windshield, any presence of a film, location of the decal, or any other interference, different electronic registration identification of motor vehicle readers/writers often have different maximum reading distances. The differences in the maximum reading distance prevents the electronic reader from determining where the corresponding vehicle enters the recognition section, that is, where it was first read. As a result, the reader cannot accurately calculate the vehicle velocity based on the distance and time it read.

Given the various interferences on the maximum read distance of the electronic registration identification of motor vehicle reader/writer, the calculation of the vehicle velocity in actual practice is often combined with the received signal strength indication (RSSI), that is, the strength of the signal returned by the electronic registration identification of the motor vehicle tag. The higher the RSSI value, the closer the vehicle is to the antenna, and vice versa. Specifically, the position of the vehicle at different times may be calculated by using the RSSI value returned from the electronic registration identification of the motor vehicle tag of the vehicle at different times, and the velocity of the vehicle may then be 
calculated according to the time differences of the corresponding positions. That is, by analyzing the RSSI value of the feedback signal that is first recognized when the corresponding vehicle enters the recognition section, it can be determined whether the vehicle position at the first recognition is at the boundary between the direct shot area and the blind area or at the farthest point in the reflection area. The formula $V=\left(S /\left(T_{o}-T_{i}\right)\right)$ can then be used to calculate the velocity of the vehicle. In actual implementation, however, the presence of blind spots and the emergence of environmental conditions may affect the results of velocity calculation. Analysis shows that although a blind spot may disappear, the radio frequency identification effect in the cross section of the blind area will still be different from that in the direct area. This is judged by the fluctuation of the number of recognitions per unit time from when the vehicle enters the recognition section to when it leaves the recognition section. If the fluctuation is not large, the vehicle has been recognized in the direct shot area the whole time. If the number of recognitions per unit time varies greatly and if one period shows a very high number of recognitions per unit time and the other period shows a relatively low number of recognitions, or if a vehicle in another lane is recognized and calculated by the electronic registration identification of the motor vehicle reader, then it is possible that a blind spot had disappeared. In a real environment, it is necessary to set up different calculation methods for calculating vehicle velocity in different situations. Since it is often difficult to accurately select the correct calculation method, the calculated vehicle velocity often deviates from the actual values.

\section{Velocity Calibration Method Based on Particle Swarm Optimization Neural Network Algorithm}

Since vehicle velocity data are affected by various environmental and equipment factors, we use the velocity data read by the OBD interface on the vehicle as the reference standard. We establish a velocity calibration model based on a particle swarm optimization neural network algorithm and incorporate various types of data influenced by environmental factors. In order to preserve the diversity of velocity data acquisition methods using electronic registration identification of motor vehicles, the source of the velocity data comes from data collected by the electronic registration identification of the motor vehicle reader/writer on vehicles in the lane of interest and in other lanes. On the two-way, eight-lane road section, the velocity of vehicles passing through the recognition section can theoretically be calculated from the data read by four electronic registration identification of motor vehicle readers/writers. This completes the detection network of electronic registration identification of motor vehicle readers/writers set up to improve the accuracy of velocity calibration. In addition, to improve the calculation efficiency of the neural network, attention is given to the distribution efficiency of sensing tasks and the collection efficiency of sensing data in the edge network in order to improve the scope of coverage and sensing efficiency of the reader application. The readers that have deployed the task distribution service will serve as the source of task distribution and continuously distribute vehicle-borne velocity detection tasks to mobile devices. During the data collection process, the vehicle-borne devices serve as the source of the velocity data detection, and the data are transmitted back to the readers involved in data collection $[17,18]$.

3.1. RBF Neural Network. The RBF neural network is an artificial neural network that uses local adjustment to perform function mapping. It has strong input and output mapping functions and is an optimal network for performing mapping functions in feed-forward networks. It has a strong nonlinear approximation capability, a simple network architecture, and a fast learning velocity. The output matrix of the hidden layer after iterative convergence has a linear relationship with the output. It is an ideal algorithm for calculating the degree of influence $[19,20]$.

Using RBF as the "base" of the hidden unit constitutes the hidden layer space, so that the input vector can be directly mapped to the hidden space, without the need for a weighted connection. After the center point of the RBF is determined, this mapping relationship is also determined. The mapping of the hidden layer space to the output space is linear, that is, the output of the network is a linear weighted sum of the output of the hidden unit, and the weights here are the tunable parameters of the network. Here, the role of the hidden layer is to map the vector from a low dimension to a high dimension, so that the linearly inseparable case of the low dimension can become linearly separable in the high dimension. Thus, the network's mapping from the input to the output is nonlinear, while the network's output is linear with respect to the adjustable parameters. The weights of the network can be solved directly from the linear equations, which greatly speed up the learning and avoids local minima.

In the two-staged learning process of the RBF neural network, unsupervised learning mainly determines the radial basis vector and normalized parameters of the Gaussian basis function of each node of the hidden layer based on the input samples; that is, it determines the center and variance of the hidden layer basis function. In the supervised learning phase, the weights of the hidden layer and the output layer are calculated using the least square method after determining the hidden layer parameters. The output of the $i$-th hidden layer of the RBF neural network is [20-22]

$$
h_{i}(t)=\phi_{i}\left(\left\|x(t)-c_{i}\right\|\right)=\exp \left(-\sum_{i=1}^{n} \frac{\left(x_{i}(t)-c_{i}\right)^{2}}{2 s_{i}^{2}}\right),
$$

where $x(t)$ is the input vector of the network at time $t, c_{i}$ is the central vector of the $i$-th unit of the hidden layer, $s_{i}$ is the shaper parameter of the Gaussian function, where $s_{i}>0$, $1 \leq i \leq L$, and $L$ is the number of nodes of the hidden layer.

The overall output of the RBF neural network is as follows: 


$$
\begin{aligned}
y & =\sum_{i=1}^{L} w_{i} h_{i}, \\
& =\sum_{i=1}^{L} w_{i} \phi_{i}\left(\left\|x(t)-c_{i}\right\|\right), \\
& =H^{T} W
\end{aligned}
$$

where $H=\left[h_{1}, h_{2}, \ldots, h_{L}\right]^{T}$ and $W=\left[w_{1}, w_{2}, \ldots, w_{L}\right]$.

For an RBF network with $k$ input nodes, $m$ output nodes, and $n$ learning samples, the error objective function is

$$
\begin{aligned}
E(n) & =\frac{1}{2} \sum_{k=1}^{n} \lambda^{n-k} \sum_{i=1}^{M} \delta_{i}(n), \\
& =\frac{1}{2} \sum_{k=1}^{n} \lambda^{n-k} \sum_{i=1}^{M}\left[\bar{y}_{i}(n)-y_{i}(n)\right]^{2}, \\
& =\lambda E(n-1)+\frac{1}{2} \sum_{i=1}^{M}\left[\bar{y}_{i}(n)-H^{T}(n) W_{i}(n-1)\right]^{2},
\end{aligned}
$$

where $\lambda$ is the forgetting factor and $\delta_{i}(n), \bar{y}_{i}(n)$, and $y_{i}(n)$ are, respectively, the error of the output node, the expected output, and the actual output.

The operation performance of the RBF neural network mainly depends on the center and radial basis of the hidden layer function and the weight of the output layer. Additionally, the number and the center of hidden nodes in the RBF network are difficult to determine, which affects the accuracy of the entire network. When the selection of the training samples contains a high degree of randomness, the number of errors will increase rapidly, and it is difficult to meet requirements. These errors will contribute directly to the calculation of the predicted results. In addition, when determining these parameters, the learning strategy of the traditional RBF neural network has a great disadvantage, that is, it can only find the optimal solution in local space. If these parameters are not set properly, the accuracy of the approximation will be reduced or the network will diverge. Furthermore, since the input samples contain various types of data, including discrete values, continuous values, and missing values, the training samples are usually obtained by random extraction. The center of the RBF neural network hidden layer basis function is selected from the input sample set, which has a large dependence on the training samples. In many cases, it is difficult to reflect the true input-output relationship of the system, and it is easy for data pathology to occur when there are too many initial center points. This sample data selection dilemma is a key problem to be solved when an RBF neural network is used for nonlinear system modeling. To this end, we introduce in this paper, a particle swarm optimization algorithm to optimize the parameters of the RBF neural network, and we apply it to the numerical calibration of vehicle velocity detection based on electronic registration identification of motor vehicle reading.
3.2. Particle Swarm Optimization Algorithm. Particle swarm optimization (PSO) is an efficient heuristic parallel search algorithm. Since it converges rapidly during optimization and does not require gradient information of the objective function, it enjoys the advantage of being simple and easy to implement, and thus has been applied in the field of engineering technology $[23,24]$.

The solution of the PSO algorithm for the optimization problem is abstracted as an entity, the "particle," without weight and volume. Each particle has a fitness value determined by the optimized function, and this value is searched in the search space at a certain velocity through the cooperation and competition among the particles. The specific process is to first initialize a group of random particles; these particles update their velocity and position by tracking the two optimal particles. The two optimal particles are the individual optimal particle (the optimal solution found by the particle itself, known as the individual optimal position) and the global optimal particle (the optimal solution found by the entire population up to now, known as the global best position).

Let the search space be an $n$-dimensional space and the particle swarm consists of $n_{s}$ particles; $x_{i}^{(t)}=$ $\left(x_{i, 1}^{(t)}, x_{i, 2}^{(t)}, \ldots, x_{i, n}^{(t)}\right)$ and $v_{i}^{(t)}=\left(v_{i, 1}^{(t)}, v_{i, 2}^{(t)}, \ldots, v_{i, n}^{(t)}\right)$ are the position and velocity, respectively, of the $i$-th particle at time $t$ in the search space, $p_{i}^{(t)}=\left(p_{i, 1}^{(t)}, p_{i, 2}^{(t)}, \ldots, p_{i, n}^{(t)}\right)$ is the personal best position (referred to as pbest) of the $i$-th particle at time $t$, and $g_{i}^{(t)}=\left(g_{i, 1}^{(t)}, p_{i, 2}^{(t)}, \ldots, g_{i, n}^{(t)}\right)$ is the global best position (referred to as gbest) at time $t$. According to the PSO algorithm, the update formula for particle velocity and position is as follows [23-25]:

$$
\begin{aligned}
& v_{i, j}^{(t+1)}=v_{i, j}^{(t)}+c_{1} r_{1}\left(p_{i, j}^{(t)}-x_{i, j}^{(t)}\right)+c_{2} r_{2}\left(g_{i}^{(t)}-x_{i, j}^{(t)}\right), \\
& x_{i, j}^{(t+1)}=x_{i, j}^{(t)}+v_{i, j}^{(t+1)},
\end{aligned}
$$

where $c_{1}$ and $c_{2}$ are the learning factors or acceleration constants, respectively, for adjusting the step size of the particle flying to the personal best position and the global best position. In order to reduce the probability of the particle to flying out of the search space, the velocity of the particle in each dimension is usually limited to a certain value within the range. Also, $r_{1}$ and $r_{2}$ are random numbers obeying a uniform distribution in the interval $[0,1]$, $i=1,2, \ldots, N_{s}, N_{s}$ is the scale of the particle swarm, and $j=1,2, \ldots, n$.

Traditional PSO algorithms generally follow the following steps to complete the iteration [26]: step one, initializing the velocity and position of the particle swarm; step two, calculating the fitness value of each particle in the particle swarm; step three, updating the personal best position of each particle; step four, updating the global best position of each particle; step five, updating the velocity and position of the particles and taking certain measures to ensure that the particles remain in the search space; step six, determining whether the termination conditions are met (terminate the algorithm if yes, or return to step two).

However, in the updating process of the traditional PSO algorithm, the inertia weight, learning factor, and maximum 
velocity jointly determine the balance between the global exploration and local development capabilities of the algorithm, which has a direct impact on the search performance of the algorithm.

\subsection{RBF Neural Network Based on Particle Swarm} Optimization. The key point of the seeking process is that the parameters of the RBF neural network are combined into a particle vector by coding, and the individual optimal value and the global optimal value of particles are reduced to the corresponding parameters of the RBF neural network after initialization; the individual utility extreme value of particles is calculated by comparison, and the individual optimal value and the global optimal value are updated after comparison with the current individual optimal value and the global optimal value. Finally, the global optimal solution is decoded, restored to the parameters of the network, and the RBF network prediction is carried out. Before neural network training, the convergence of the RBF neural network can be ensured by normalizing all sample data.

By combining the calibration requirement vehicle velocity detection data using electronic registration identification of motor vehicles with characteristics of PSO and the RBF neural network, we arrived at the following procedure for optimizing the kernel function parameter of the RBF neural network with the PSO algorithm:

Step 1. Data Normalization Processing. In order to improve the generalization ability, the convergence velocity, the correlation between influencing factors, and the fitting effect of the neural network, we use a maximum and minimum value method to normalize the sample data to the $[0,1]$ range: $y(k)=(x(k)-$ $\min (x(n)) / \max (x(n))-\min (x(n))), k=1,2, \ldots, N$, where $\min (x(n))$ is the minimum value of the sample data and $\max (x(n))$ is the maximum value of the sample data.

Step 2. The particle swarm and the neural network are initialized, the scale is determined, i.e., the dimension of the particle swarm, and the mapping is established between the dimensional space of the PSO particles and the connection weight of the neural network. The population size is set as $N_{s}$ and the maximum number of iterations as $T_{\max }$. The population location matrix $\operatorname{Rand}(x)$ and the velocity matrix $\operatorname{Rand}(v)$ are randomly generated as follows:

$$
\begin{aligned}
\operatorname{Rand}(x) & =\left[\begin{array}{ccc}
x_{1,1} & \cdots & x_{1, M} \\
\vdots & & \vdots \\
x_{N, 1} & \cdots & x_{N, M}
\end{array}\right], \\
\operatorname{Rand}(v) & =\left[\begin{array}{ccc}
v_{1,1} & \cdots & v_{1, M} \\
\vdots & & \vdots \\
v_{N, 1} & \cdots & v_{N, M}
\end{array}\right], \\
x_{i, j}^{(t+1)} & =x_{\min }^{(t)}+\left(x_{\max }^{(t)}-x_{\min }^{(t)}\right) \times \operatorname{Rand}(x),
\end{aligned}
$$

$$
v_{i, j}^{(t+1)}=-v_{\max }^{(t)}+2 v_{\max }^{(t)} \times \operatorname{Rand}(v),
$$

where $M=k(n+1), k$ is the number of nodes in the hidden layer, $n$ is the dimension of the input feature vector, $\left\{x_{i, j}|i=1,2, \ldots, N ; j=1,2, \ldots, M|\right\}$ represents the $j$-th dimension component of the $i$-th particle position vector, $\left(x_{i, 1}, x_{i, 2}, \ldots, x_{i, M}\right)$ corresponds to the radial basis parameter of the RBF neural network, representing the $j$-th dimension component of the velocity vector of the $i$-th particle, and the value of $v_{\max }^{(t)}$ is related to $x_{\max }^{(t)}$ and $x_{\min }^{(t)}$.

Step 3. The fitness of each particle is calculated, the mean square error of the neural network is used as the fitness function of PSO. The fitness function is a measure of the position of particles in space. The greater the value of the fitness function, the better the position of the particle. It is defined as follows:

$$
\text { FitnessFunc }_{i}=\frac{1}{1+D\left(x_{i, 1}, x_{i, 2}, \ldots, x_{i, M}\right)},
$$

where FitnessFunc ${ }_{i}$ is the fitness function value of the $i$-th particle and $D\left(x_{i, 1}, x_{i, 2}, \ldots, x_{i, M}\right)$ is the sum of the average errors squared of the neural network when the kernel function parameter is equal to $\left(x_{i, 1}, x_{i, 2}, \ldots, x_{i, M}\right)$.

Step 4. For each particle, its fitness is compared with the fitness of the best location it has experienced, and if the former is better, pbest is updated.

Step 5. For each particle, its fitness is compared with the fitness of the best location experienced by the swarm, and if the former is better, gbest is updated.

Step 6. The velocity and position of the particles are updated, and dynamic adaptive adjustment is performed using the inertial weights. Steps 3 through 5 are repeated until the calculation requirements are met. When the algorithm iteration stops, the weights and thresholds of the neural network correspond to the global optimum, which is the optimal solution to the problem.

Step 7. The vehicle velocity data are calibrated using the trained RBF neural network while simultaneously assessing the accuracy of the velocity data.

3.4. Numerical Optimization of Multireader Velocity Data Using Mobile Edge Computing. The shortcomings of the RBF neural network, including the local optima and the effects on PSO by inertial weight, learning factor, and maximum velocity, have direct impact on the calibration results in the probability sense. In order to further improve the accuracy of velocity calibration, the capability of having multiple readers simultaneously implementing velocity detection of the same electronic registration identification of the motor vehicle tag had been achieved before our study was conducted. After each reader/writer calibrates the velocity data using the RBF neural network based on the particle swarm 
optimization algorithm, the k-means method is used to find the cluster center value of different reader/writer values as the final velocity calibration value. The calculation method is as follows.

Assuming that the cluster partitioning is $\left(c_{1}, c_{2}, \ldots, c_{k}\right)$, the goal is to minimize the squared error $E$ :

$$
E=\sum_{i=1}^{k} \sum_{x \in C_{i}}\left\|x-\mu_{i}\right\|^{2}
$$

where $\mu_{i}$ is the mean vector of cluster $C_{i}$ and $\mu_{i}=\left(1 /\left|C_{i}\right|\right) \sum_{x \in C_{i}} x$.

In order to improve the computing efficiency of the reader/writer, the emphasis of the optimization algorithm is placed on the distribution efficiency of the sensing tasks and the collection efficiency of the sensing data in the edge network of the reader/writer. By designating a certain reader/writer as the main computing reader/writer, readers/ writers already deployed for task distribution services will serve as sources for task distribution in the task distribution computation process. The main computing reader/writer will continually distribute the vehicle terminal velocity detection computing tasks to different readers. During the data collection and optimization process, all the readers/ writers serve as the source of vehicle-borne devices in acquiring data and return the data of completed computing tasks to the main computing reader/writer for final calculation.

\section{Tests and Results}

4.1. Test Method and Data Description. The tests were conducted in a road section where the electronic registration identification of the motor vehicle reader has already been installed for testing. The road was a two-way, eight-lane urban expressway, with a gantry equipped with internetconnected testing instruments. There were eight sets of electronic registration identification of the motor vehicles readers, with each traffic lane aligned with at least one reader for direct acquisition of the electronic registration identification of motor vehicle data in the vertical direction.

Since the automobile electronic registration identification of the motor vehicle reader has already acquired the capability of group acquisition, data can be collected simultaneously when multiple electronic registration identification of motor vehicles pass through at the same time, and we placed 1000 electronic registration identification of motor vehicles simultaneously on the test vehicles and scattered them at different locations inside the vehicles. In this manner, we demonstrated the interference immunity performance between the electronic registration identification of motor vehicles.

The data read by the OBD interface on the vehicle is the true velocity of the moving vehicle. With the temporal data of the OBD interface synchronized with the temporal data of the reader, we selected three sedans as test vehicles, each carrying 1000 automotive electronic registration identification of motor vehicle cards, to pass under the gantry's readers with different velocity. The sample data set was formed with the data read from the 1000 vehicle-borne electronic registration identification of motor vehicles as the input, with the actual velocity data read by the OBD interface as the output, all matched with the time data. It should be noted that when the vehicles passed under the gantry, the velocity data of the electronic registration identification of motor vehicles in the vehicles were read by multiple readers, and each electronic registration identification of motor vehicles can form velocity data in different readers. The final complete sample data set was obtained by testing the three sedans in different lanes at different velocity, and with the data from repeated tests matched. A comparison of the true values of the velocity and the measured values of the velocity is shown in Figure 3. The abscissa is for the measured value of the velocity and the ordinate is for the true value of the velocity. If the two values are the same, the point falls on the straight line of $y=x$. If the true value is less than the measured value, the point falls below the straight line, and vice versa.

Based on the data distribution shown in Figure 3, the differences between the true velocity and the measured velocity are shown in Figure 4.

4.2. Test Results and Analysis. The experimental results show that the best effect is that the number of hidden layer centers of the RBF neural network is 9, and the network structure is 4-12-1. PSO algorithm parameters are divided into $c_{1}=c_{2}=2.0$, the number of particle population is 46 , and the maximum times of iterations is set to 500 . Before neural network training, the convergence of the RBF neural network can be ensured by normalizing all sample data. After many experiments, when the control accuracy is 0.005 , the average times of iterations using only the RBF neural network is about 320, while the average times of iterations using the PSO-RBF neural network is about 95.

The errors in the collected detection data reflects the difference between the data collected by the traditional velocity detection method and the true values. Most of the data had an error rate within $5 \%$, which can fully meet the needs of practical applications; to a certain extent, this is better than the error rate of video, geomagnetic, and other methods for collecting velocity data and is closer to the needs of practical applications. However, in some industries or specific applications, detected data need to be closer to the actual velocity, and higher requirements for controlling the data error rate are imposed. In this paper, we use the particle swarm optimization neural network method to calibrate the velocity detected with electronic registration identification of motor vehicles so as to obtain a detected velocity that is closer to the actual data.

Test data for different velocity and different lanes were screened for perfect time matches; a total of 11086 sets of complete data were obtained. The data acquired for the eight lanes showed an approximately uniform distribution. It should be noted that since the driver can only control the velocity at a certain interval when driving the vehicle through the gantry and the velocity on urban expressways is not less than $20 \mathrm{~km} / \mathrm{h}$, all data values below $20 \mathrm{~km} / \mathrm{h}$ were 


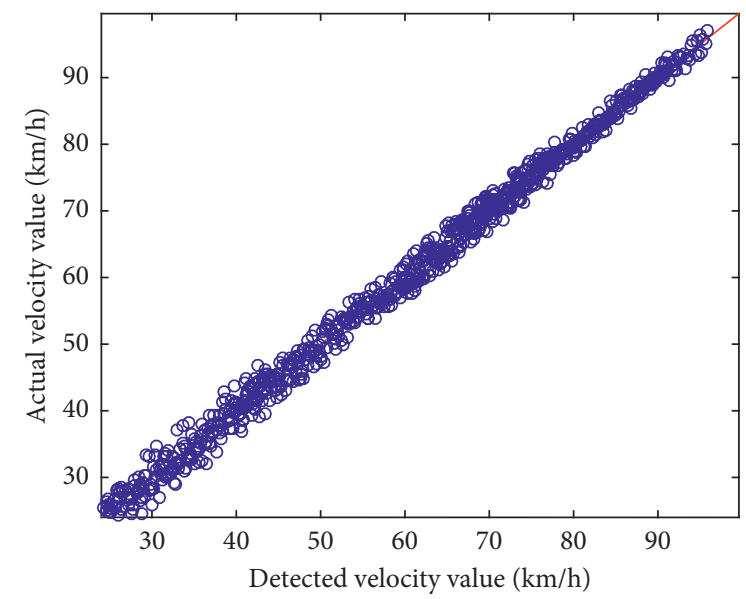

Figure 3: Actual velocity value and the detected velocity value.

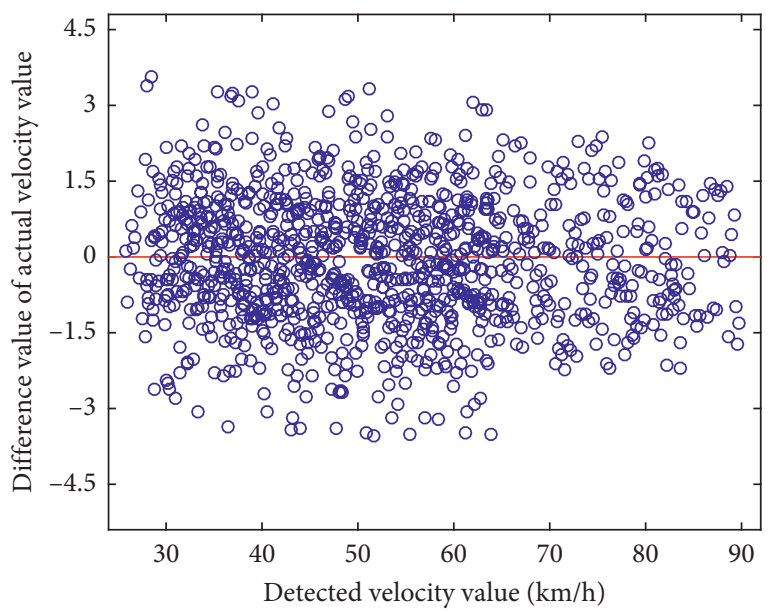

Figure 4: The error distribution.

discarded. In the meantime, requirements for safe driving forbids drivers from driving over $100 \mathrm{~km} / \mathrm{h}$ under normal circumstances (although the electronic registration identification of motor vehicle readers/writers can accurately detect velocities up to $180 \mathrm{~km} / \mathrm{h}$ ), and therefore no tests were carried out over $100 \mathrm{~km} / \mathrm{h}$.

Using the same display method as in Figure 3, the actual velocity and the detected velocity are displayed for four lanes of the selected side of the road (Figure 5). Figures 5(a)-5(d) show, respectively, the velocity values of vehicles on the four lanes. The lane in Figure 5(a) is the closest to the center line of the road.

It can be seen from Figure 5 that, after the detection position of the electronic registration identification of the motor vehicle reader is fixed on the gantry, the detection results are also relatively fixed; detection in different lanes would not cause a large difference. However, since all the velocity values are detected by hardware, the velocity calculation method belongs to a generic algorithm. As long as its error is within a preset range, it meets the user requirement. For this reason, the error rate is still too high for certain specific applications. In addition, the distribution results of the detected velocity also showed that the errors of the two lanes near the center line and the emergency lane are slightly higher than that of the middle two lanes. It can also be seen that the faster the velocity, the lower the error. And, the errors are slightly larger at lower velocities.

The tests are conducted on a selected middle lane, and the velocity detection results are calibrated using the following methods. The RBF neural network is based on particle swarm optimization, and multireader/writer velocity values are optimized with the RBF neural network based on PSO. The calibration results are shown in Figures 6-9.

As can be seen from Figures 6-9, the optimal output calibration result is the value of the multireader velocity with numerical optimization based on the particle swarm-optimized RBF neural network. Especially in the low-velocity stage, the detected velocity value can be better calibrated, and the velocity error value can be further reduced. The four scatter plots above show that, in the $25-40 \mathrm{~km} / \mathrm{h}$ range, the detected velocity values and the actual velocity values are greater than those in other regions. The mean square error value for all data is 0.0965 , and the Pearson coefficient $r=\left(\sum\left(x_{i}-\bar{x}\right)\left(y_{i}-\bar{y}\right) / \sqrt{\sum\left(x_{i}-\bar{x}\right)^{2}} \sqrt{\sum\left(y_{i}-\bar{y}\right)^{2}}\right)$, a measure of correlation, is calculated to be 0.9301 .

In order to further compare the differences in the calibration process of velocity detection in different lanes, we statistically analyzed all the test data. The statistical results are shown in Table 1. It should be noted that in the second column of the table, labeled "optimization algorithm," "uncalibrated" represents data obtained without calibration, "Algorithm 1" represents the detected velocity output value calibrated by the RBF neural network, "Algorithm 2" represents the detected velocity output value calibrated by the RBF neural network based on particle swarm optimization, and "Algorithm 3" represents the detected velocity output value calibrated by the RBF neural network based on particle swarm optimization and on multireader velocity numerical optimization.

Table 1 lists the calibration results of the detected velocity values for different lanes using different algorithms. As far as the trend of calibration accuracy is concerned, the accuracy of the data from the one-way middle lane is relatively high. For detected velocity values calibrated with multireader numerical optimization based on the PS-optimized RBF neural network, the percentages of data with errors less than or equal to $5 \%$ are $92.39 \%, 92.10 \%, 91.41 \%$, and $91.11 \%$ for lane nos. 2, 3, 6, and 7, respectively. The average percentage of data with errors less than or equal to $5 \%$ is $91.76 \%$ for the middle lane, $82.13 \%$ for the edge lane, and $87.12 \%$ for all lanes combined. In terms of actual applications, results with an error rate less than $10 \%$ have an application value, and the calibrated velocity detection data have met this requirement. A lower error rate not only is indicative of the method's application value, but also reflects the real traffic condition more realistically. In the comprehensive statistical analysis of the error rate of the detected velocity value, the rate for the middle lane is lower than that for the edge lane. After calibration, the error values for different testing velocities are statistically consistent. 


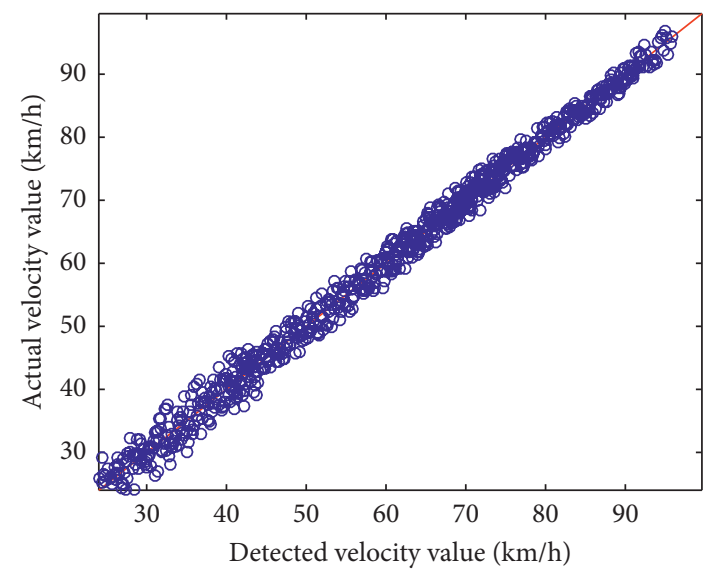

(a)

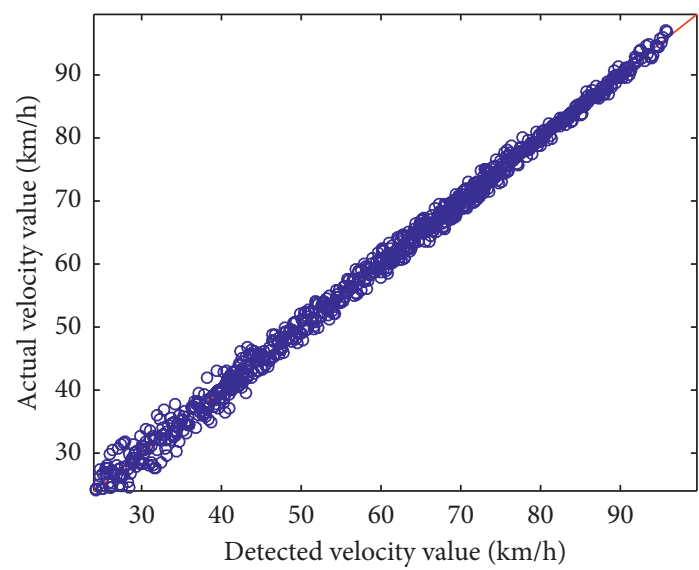

(c)

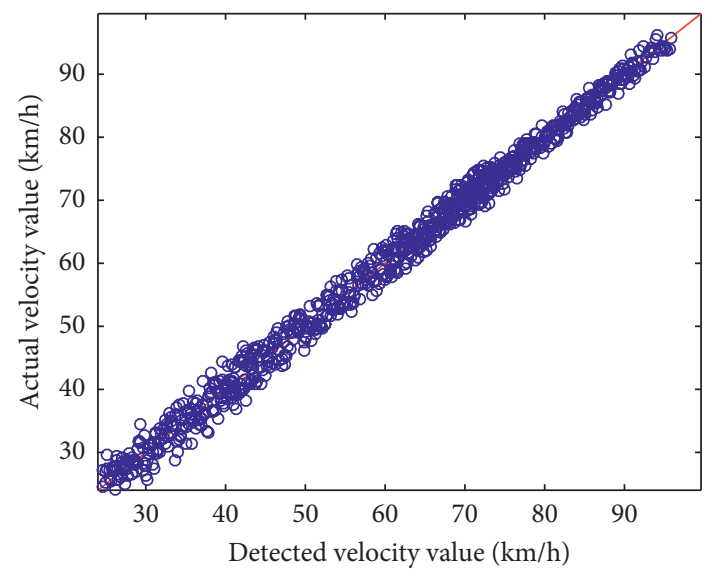

(b)

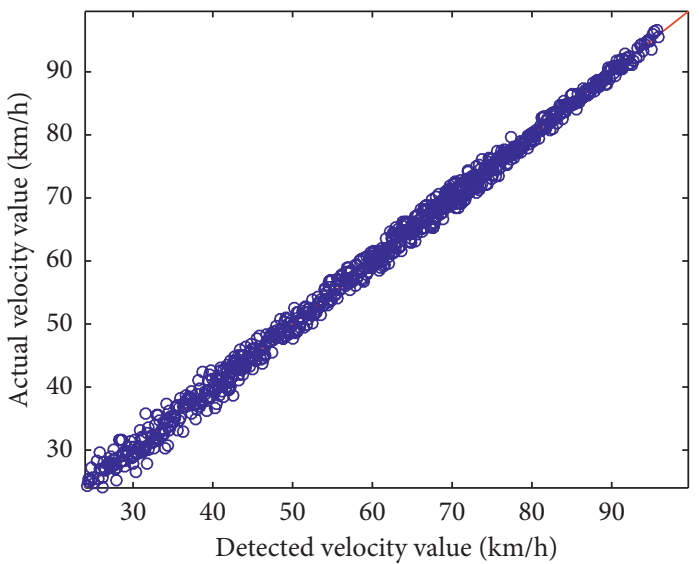

(d)

FIgURE 5: Actual velocity value and the detected velocity value for four different lanes.

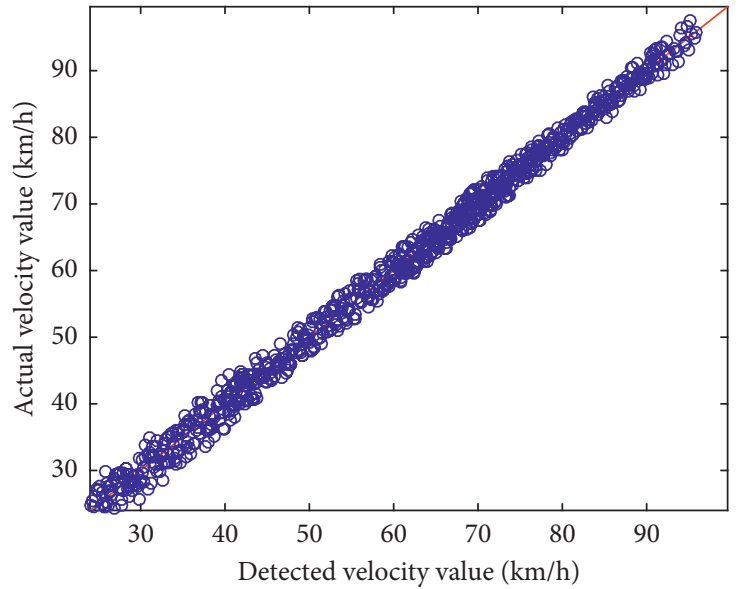

Figure 6: Actual velocity value and the detected velocity value for one lane.

We calculated the mean square error and the Pearson coefficient of the detected and calibrated velocity values with multireader numerical optimization based on the PS-optimized RBF neural network. From lane nos. 1 through 8 , the mean square error values are 0.0856 , $0.0653,0.0623,0.0841,0.0901,0.0679,0.0688$, and
0.0831, respectively; the corresponding Pearson coefficients are $0.9116,0.9412,0.9332,0.9193,0.9113,0.9401$, 0.939 , and 0.9128 , respectively. The mean square error value and the Pearson coefficient value both demonstrate that the velocity calibration value in the middle lane is accurate. 


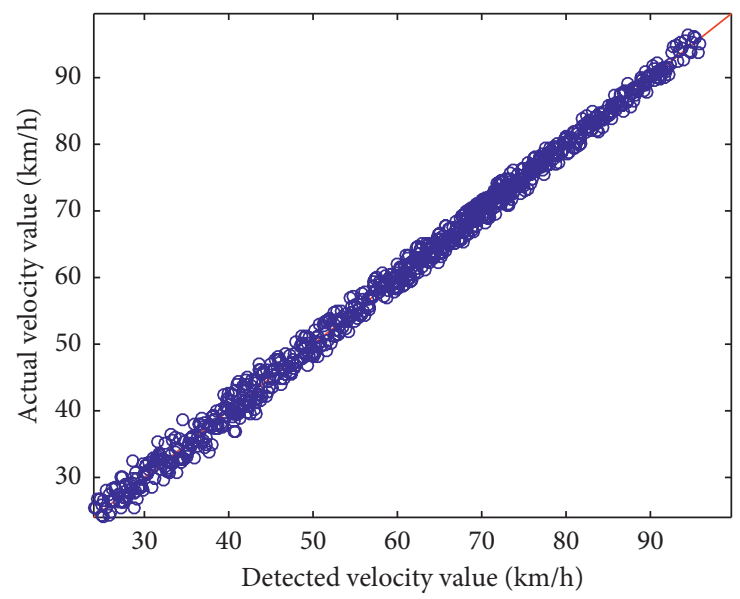

FIGURE 7: Actual velocity value and the detected velocity value calibrating based on the RBF neural network.

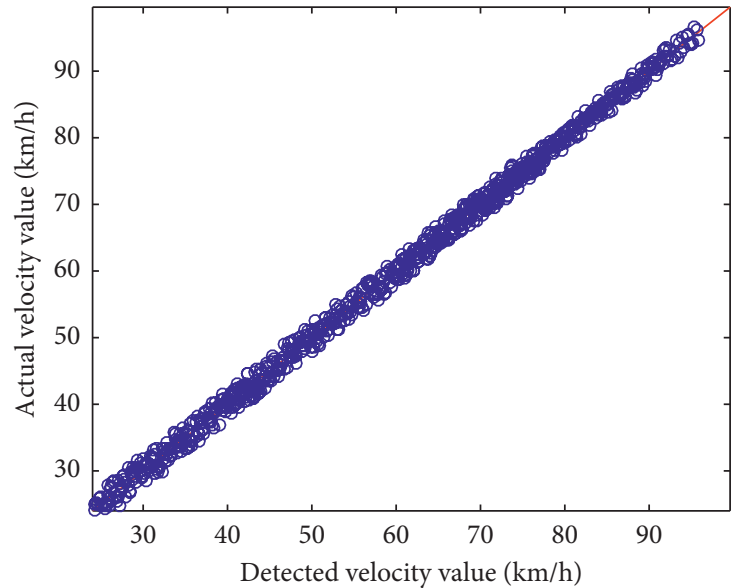

FIGURE 8: Actual velocity value and the detected velocity value calibrating based on the RBF neural network based on particle swarm optimization.

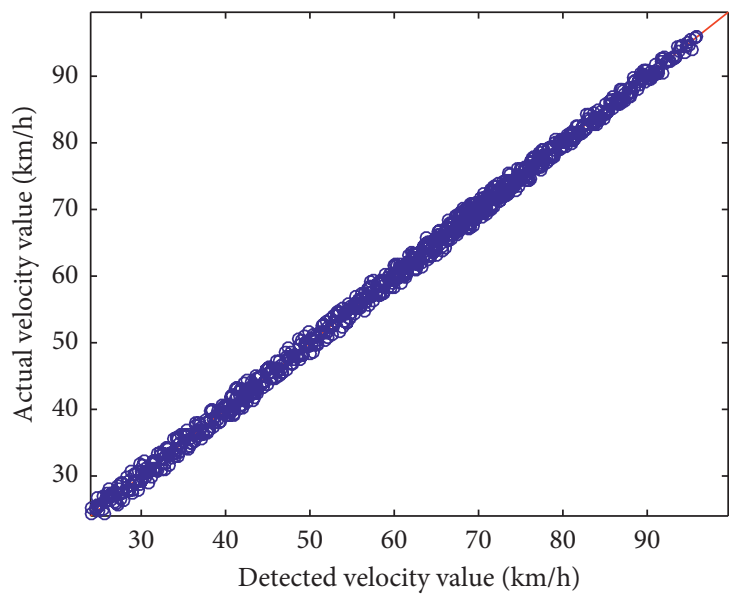

FIGURE 9: Actual velocity value and the detected velocity value calibrating based on numerical optimization of multireader velocity based on the particle swarm-optimized RBF neural network.

To further analyze the test results, we plot in Figure 10 the error values of the uncalibrated detected velocity values and compare them with the error values of the calibrated detected velocity values using multireader numerical optimization based on the particle swarm optimization of the RBF neural network. The blue circle represents the error 
TABLE 1: The calibration results of detected velocity values under different algorithms.

\begin{tabular}{|c|c|c|c|c|c|c|c|c|c|}
\hline $\begin{array}{l}\text { Lane } \\
\text { number } \\
\text { (number } \\
\text { of } \\
\text { samples) }\end{array}$ & $\begin{array}{l}\text { Optimization } \\
\text { method }\end{array}$ & $\begin{array}{l}\text { Number } \\
\text { of samples } \\
\text { with error } \\
\leq 1 \%\end{array}$ & $\begin{array}{c}\text { Proportion } \\
\text { of samples } \\
\text { with error } \\
\quad \leq 1 \%\end{array}$ & $\begin{array}{c}\text { Number } \\
\text { of samples } \\
\text { with error } \\
\leq 3 \%\end{array}$ & $\begin{array}{c}\text { Proportion } \\
\text { of samples } \\
\text { with error } \\
\leq 3 \%\end{array}$ & $\begin{array}{l}\text { Number } \\
\text { of samples } \\
\text { with error } \\
\leq 5 \%\end{array}$ & $\begin{array}{c}\text { Proportion } \\
\text { of samples } \\
\text { with error } \\
\leq 5 \%\end{array}$ & $\begin{array}{c}\text { Number } \\
\text { of samples } \\
\text { with error } \\
>5 \%\end{array}$ & $\begin{array}{c}\text { Proportion } \\
\text { of samples } \\
\text { with error } \\
\quad>5 \%\end{array}$ \\
\hline \multirow{4}{*}{$\begin{array}{l}\text { Lane no. } 1 \\
(1256)\end{array}$} & Uncalibrated & 284 & 22.61 & 539 & 42.91 & 798 & 63.54 & 458 & 36.46 \\
\hline & Algorithm 1 & 302 & 24.04 & 674 & 53.66 & 878 & 69.90 & 378 & 30.10 \\
\hline & Algorithm 2 & 332 & 26.43 & 699 & 55.65 & 909 & 72.37 & 347 & 27.63 \\
\hline & Algorithm 3 & 386 & 30.73 & 759 & 60.43 & 1053 & 83.84 & 203 & 16.16 \\
\hline \multirow{4}{*}{$\begin{array}{l}\text { Lane no. } 2 \\
(1432)\end{array}$} & Uncalibrated & 332 & 23.18 & 683 & 47.70 & 995 & 69.48 & 437 & 30.52 \\
\hline & Algorithm 1 & 399 & 27.86 & 796 & 55.59 & 1092 & 76.26 & 340 & 23.74 \\
\hline & Algorithm 2 & 448 & 31.28 & 807 & 56.35 & 1245 & 86.94 & 187 & 13.06 \\
\hline & Algorithm 3 & 498 & 34.78 & 893 & 62.36 & 1323 & 92.39 & 109 & 7.61 \\
\hline \multirow{4}{*}{$\begin{array}{l}\text { Lane no. } 3 \\
\text { (1494) }\end{array}$} & Uncalibrated & 353 & 23.63 & 698 & 46.72 & 1002 & 67.07 & 492 & 32.93 \\
\hline & Algorithm 1 & 387 & 25.90 & 769 & 51.47 & 1104 & 73.90 & 390 & 26.10 \\
\hline & Algorithm 2 & 472 & 31.59 & 821 & 54.95 & 1265 & 84.67 & 229 & 15.33 \\
\hline & Algorithm 3 & 531 & 35.54 & 901 & 60.31 & 1376 & 92.10 & 118 & 7.90 \\
\hline \multirow{4}{*}{$\begin{array}{l}\text { Lane no. } 4 \\
\text { (1379) }\end{array}$} & Uncalibrated & 301 & 21.83 & 592 & 42.93 & 879 & 63.74 & 500 & 36.26 \\
\hline & Algorithm 1 & 344 & 24.95 & 673 & 48.80 & 953 & 69.11 & 426 & 30.89 \\
\hline & Algorithm 2 & 380 & 27.56 & 733 & 53.15 & 1034 & 74.98 & 345 & 25.02 \\
\hline & Algorithm 3 & 413 & 29.95 & 853 & 61.86 & 1143 & 82.89 & 236 & 17.11 \\
\hline \multirow{4}{*}{$\begin{array}{l}\text { Lane no. } 5 \\
(1402)\end{array}$} & Uncalibrated & 267 & 19.04 & 582 & 41.51 & 949 & 67.69 & 453 & 32.31 \\
\hline & Algorithm 1 & 309 & 22.04 & 672 & 47.93 & 1012 & 72.18 & 390 & 27.82 \\
\hline & Algorithm 2 & 350 & 24.96 & 749 & 53.42 & 1044 & 74.47 & 358 & 25.53 \\
\hline & Algorithm 3 & 401 & 28.60 & 829 & 59.13 & 1097 & 78.25 & 305 & 21.75 \\
\hline \multirow{4}{*}{$\begin{array}{l}\text { Lane no. } 6 \\
(1386)\end{array}$} & Uncalibrated & 341 & 24.60 & 603 & 43.51 & 1015 & 73.23 & 371 & 26.77 \\
\hline & Algorithm 1 & 422 & 30.45 & 692 & 49.93 & 1098 & 79.22 & 288 & 20.78 \\
\hline & Algorithm 2 & 478 & 34.49 & 795 & 57.36 & 1164 & 83.98 & 222 & 16.02 \\
\hline & Algorithm 3 & 503 & 36.29 & 832 & 60.03 & 1267 & 91.41 & 119 & 8.59 \\
\hline \multirow{4}{*}{$\begin{array}{l}\text { Lane no. } 7 \\
(1429)\end{array}$} & Uncalibrated & 332 & 23.23 & 586 & 41.01 & 998 & 69.84 & 431 & 30.16 \\
\hline & Algorithm 1 & 441 & 30.86 & 689 & 48.22 & 1086 & 76.00 & 343 & 24.00 \\
\hline & Algorithm 2 & 476 & 33.31 & 807 & 56.47 & 1239 & 86.70 & 190 & 13.30 \\
\hline & Algorithm 3 & 513 & 35.90 & 841 & 58.85 & 1302 & 91.11 & 127 & 8.89 \\
\hline \multirow{4}{*}{$\begin{array}{l}\text { Lane no. } 8 \\
(1308)\end{array}$} & Uncalibrated & 290 & 22.17 & 490 & 37.46 & 878 & 67.13 & 430 & 32.87 \\
\hline & Algorithm 1 & 304 & 23.24 & 572 & 43.73 & 954 & 72.94 & 354 & 27.06 \\
\hline & Algorithm 2 & 343 & 26.22 & 689 & 52.68 & 1003 & 76.68 & 305 & 23.32 \\
\hline & Algorithm 3 & 376 & 28.75 & 772 & 59.02 & 1097 & 83.87 & 211 & 16.13 \\
\hline
\end{tabular}

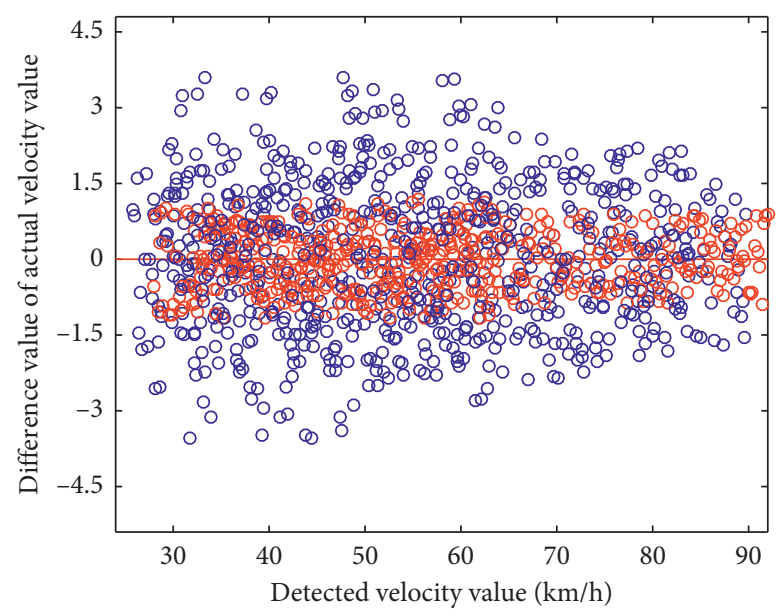

Figure 10: The calibration error distribution. 
value of the uncalibrated detected velocity values and the red circle represents the error of the detected velocity values calibrated by the RBF neural network based on particle swarm optimization after multireader numerical optimization.

The scatter plot of the error distribution for the uncalibrated and calibrated detected velocity values shows that the algorithm proposed here can achieve accurate calibration of the velocity detected by electronic registration identification of motor vehicles and that the error value can be controlled within an acceptable range. In actual application, if the various influencing factors are fully considered, including the external environment, electromagnetic interference, windshield material, angle, different vehicle types, presence of film, decal placement location, and other interference factors, then the test results can accurately reflect the value of the real velocity, and the algorithm can provide data even closer to the real situation of practical application scenarios for improving traffic management efficiency such as in traffic rule enforcement, traffic status detection, traffic congestion index calculation, and navigation and parking guidance.

\section{Conclusion}

Based on vehicle velocity values collected by electronic registration identification of motor vehicles and by on-board (OBD) equipment and combined with actual application needs, we calibrated the detected velocity output value of electronic registration identification of motor vehicles using an RBF neural network, particle swarm optimization-based RBF neural network, and multireader numerical optimization based on a particle swarm-optimized RBF neural network and compared the data with that data collected by OBD. We then analyzed the errors. The method proposed here can greatly improve the accuracy of the velocity detected by the electronic registration identification of motor vehicles and make it closer to the real value through the use of an algorithm. The test results show that the results predicted by the method can more accurately reflect the actual driving velocity. The proposed method can be an important reference for improving the manufacture and application of electronic registration identification of motor vehicle software and hardware. In addition, since the two methods of data collection using electronic registration identification of motor vehicles and using on-board (OBD) devices both have their limitations, such as data acquisition cycle and time matching, the method proposed here is established on the velocity values selected from the same data by time matching. As a result, part of the time series data is incomplete, and less than $30 \%$ of all the data collected are used as test samples. With only $30 \%$ of the data, it is difficult to fit or predict velocity values using the time series method, and thus the data analyzed in this paper are slightly lacking in the time dimension. This would be one area to strengthen in future studies, and the focus should be on the fitting of velocity data with different time stamps. The fitted velocity detection data, together with the algorithm described here, would then be able to completely describe the vehicle trajectory and provide important guidance for algorithm selection and model optimization.

\section{Data Availability}

The original data used to support the findings of this study are restricted by the relevant law enforcement departments in order to protect vehicle information privacy and law enforcement basis. Data are available from relevant law enforcement departments for researchers who meet the criteria for access to confidential data.

\section{Conflicts of Interest}

The authors declare that they have no conflicts of interest.

\section{Acknowledgments}

This research was funded by the National Key Research and Development Program (nos. 2017YFD0700602, 2018YFB2003500, and 2018YFB1700200) and the Key Research and Development Plan of Shaanxi Province (2018ZDXM-GY-041).

\section{References}

[1] X. Gu, J. Hu, B. Qian et al., "Application and study on electronic identification of motor vehicles in intelligent transport," Journal of Highway and Transportation Research and Development, vol. 34, no. S2, pp. 110-117, 2017.

[2] H. Jiang, W. Fang, and J. Huang, "Design of parking management system based on the electronic registration identification," China Science and Technology Information, vol. 28, no. 23, pp. 78-79, 2016.

[3] R. Wu and H. Yang, "Application and research of the electronic registration identification in traffic management field," Police Technology, vol. 7, no. 3, pp. 24-26, 2017.

[4] Y. Tao, X. Yan, T. Wang et al., "Intelligent transportation system development strategy for future intelligent society," Science \& Technology Review, vol. 37, no. 34, pp. 48-53, 2016.

[5] W. Lang, S. Tian, Schwarcz et al., "A review of application of driving simulation technology in R\&D of vehicular intelligence technology," Journal of Highway and Transportation Research and Development, vol. 34, no. 12, pp. 140-150, 2017.

[6] C. Zuo, Z. You, and X. Niu, "The application of the electronic registration identification in public security traffic management," Transportation Enterprise Management, vol. 32, no. 1, pp. 91-93, 2017.

[7] Z. L. Sun, "The application and practice of the electronic registration identification," China Information Security, vol. 37, no. 10, pp. 68-70, 2016.

[8] J. Knowles and D. Corne, "Properties of an adaptive archiving algorithm for storing nondominated vectors," IEEE Transactions on Evolutionary Computation, vol. 7, no. 2, pp. 100116, 2003.

[9] K. Deb, M. Mohan, and S. Mishra, "Evaluating the $\varepsilon$-domination based multi-objective evolutionary algorithm for a quick computation of pareto-optimal solutions," Evolutionary Computation, vol. 13, no. 4, pp. 501-525, 2005.

[10] M. Laumanns, L. Thiele, K. Deb, and E. Zitzler, "Combining convergence and diversity in evolutionary multiobjective optimization," Evolutionary Computation, vol. 10, no. 3, pp. 263-282, 2002. 
[11] M. Wu, S.-L. Dai, and C. Yang, "Mixed reality enhanced user interactive path planning for omnidirectional mobile robot," Applied Sciences, vol. 10, no. 3, p. 1135, 2020.

[12] J. Luo, Z. Lin, Y. Li, and C. Yang, "A teleoperation framework for mobile robots based on shared control," IEEE Robotics and Automation Letters, vol. 5, no. 2, pp. 377-384, 2020.

[13] H. Kong, C. Yang, G. Li, and S.-L. Dai, "A sEMG-based shared control system with no-target obstacle avoidance for omnidirectional mobile robots," IEEE Access, vol. 8, no. 1, pp. 26030-26040, 2020.

[14] W. F. Leong and G. G. Yen, "PSO-based multiobjective optimization with dynamic population size and adaptive local archives," IEEE Transactions on Systems, Man, and Cybernetics, Part B (Cybernetics), vol. 38, no. 5, pp. 1270-1293, 2008.

[15] S. Yang, M. Li, X. Liu, and J. Zheng, "A grid-based evolutionary algorithm for many-objective optimization," IEEE Transactions on Evolutionary Computation, vol. 17, no. 5, pp. 721-736, 2013.

[16] L. Li, "Ranking-based multi-objective particle swarm optimization: research and application," Doctoral Dissertation of Zhejiang University of Technology, 2017.

[17] W. Li, C. Yang, Y. Jiang, X. Liu, and C.-Y. Siu, "Motion planning for omni-directional wheeled mobile robot by potential field method," Journal of Advanced Transportation, vol. 2017, Article ID 4961383, 11 pages, 2017.

[18] D. Zhao, H. Ma, S. Tang, and X.-Y. Li, "Coupon: a cooperative framework for building sensing maps in mobile opportunistic networks," IEEE Transactions on Parallel and Distributed Systems, vol. 26, no. 2, pp. 392-402, 2015.

[19] J. Yang, N. Zhang, M. Li et al., "Vehicle information influence degree screening method based on GEP optimized RBF neural network," Complexity, vol. 2018, Article ID 1067927, 12 pages, 2018.

[20] L. Wang, J. Yang, N. Zhang et al., “Time-space relationship analysis model on the bus driving characteristics of different drivers based on the traffic performance index system," Tehnicki Vjesnik-Technical Gazette, vol. 25, no. 1, pp. 236-244, 2018.

[21] C. Yang, C. Chen, W. He, R. Cui, and Z. Li, "Robot learning system based on adaptive neural control and dynamic movement primitives," IEEE Transactions on Neural Networks and Learning Systems, vol. 30, no. 3, pp. 777-787, 2019.

[22] J. Luo, C. Yang, N. Wang, and M. Wang, "Enhanced teleoperation performance using hybrid control and virtual fixture," International Journal of Systems Science, vol. 50, no. 3, pp. 451-462, 2019.

[23] J. Kennedy and R. Eberhart, "Particle swarm optimization," in Proceedings of the International Conference on Neural Networks, vol. 4, pp. 1942-1948, IEEE, Perth, WA, Australia, December 1995.

[24] E. Zhang, "Research on multi-objective particle swarm optimization algorithm and applications," Doctoral Dissertation of Nanjing University University of Science and Technology, 2016.

[25] J. Luo, C. Liu, Y. Feng, and C. Yang, "A method of motion recognition based on electromyographic signals," Advanced Robotics, vol. 35, pp. 1-9, 2020.

[26] X. Li, "Niching without niching parameters: particle swarm optimization using a ring topology," IEEE Transactions on Evolutionary Computation, vol. 14, no. 1, pp. 150-169, 2010. 